\title{
Application of Peak Nasal Inspiratory Flow reference values in the treatment of allergic rhinitis*
}

\section{Silvia de Souza Campos Fernandes, Claudia Ribeiro De Andrade, Cássio da Cunha Ibiapina}

Department of Pediaetrics, Faculty of Medicine, Federal University of Minas Gerais, Belo Horizonte, Minas Gerais, Brazil
Rhinology 52: 133-136, 2014

DOl:10.4193/Rhino13.158

*Received for publication:

October 6,2013

Accepted: December 10, 2013

\begin{abstract}
Objective: To assess the applicability of the Peak Nasal Inspiratory Flow (PNIF) curves in follow-up of children in the treatment of allergic rhinitis.
\end{abstract}

Methods: Prospective study of 40 patients with AR, grouped in corticosteroid spray versus physiological saline solution use. Follow up for 10 weeks through clinical score and PNIF percentages in relation to the reference curves, with was-out at week 8 . Statistical assessment of the effect of treatment on variation of PNIF and clinical score was calculated by ANOVA model and Multiple Comparison of Means Test - Least Significant Difference.

Results: There was a statistically significant influence of the group, time and interaction between time and group on PNIF percentages. Throughout follow up, patients from the treatment group had mean PNIF percentages significantly higher than the placebo group. Clinical score results also demonstrated a statistically significant influence between the groups, time and interaction between time and group.

Conclusion: Increase in PNIF percentage values observed in children treated with intranasal corticosteroids revealed the applicability of PNIF curves in their follow up.

Key words: rhinitis, flow, score, children, reference values

\section{Introduction}

Allergic rhinitis (AR) is an important public health problem, due to its high prevalence and impact on patients' productivity at work, lives and school performance ${ }^{(1)}$. Symptoms include nasal obstruction, runny nose, nasal itching and sneezing. Its prevalence is increasing worldwide, with rates between $2.2 \%$ and $27.3 \%$ in children from 6 to 7 years of age, and from $4.5 \%$ to $45.5 \%$, in those aged from 13 to $14^{(2-6)}$. AR diagnosis is clinical, based on signs, symptoms and evaluation by anterior rhinoscopy. Clinical scores help both diagnosis and monitoring of patients ${ }^{(7)}$. Nasal obstruction constitutes a classic symptom of $A R$, however, its quantification is difficult by clinical examination, demanding objective measurements.
Regarding objective measurements, studies are still scarce, particularly in children, with a growing interest in obtaining parameters compatible with the pediatric age, avoiding use of absolute values and extrapolation of adult values. Recently, three international groups developed peak nasal inspiratory flow (PNIF) reference values for healthy children and adolescents ${ }^{(8-11)}$. For the Brazilian population, reference values for children and adolescents between 8 and 15 years of age were proposed (8). However, studies on their applicability in AR patients treated with intranasal corticosteroids were not found. This paper aims to assess the applicability of the PNIF curves in children and adolescents with AR treated with intranasal corticosteroids. 


\section{Materials and methods}

A prospective study was performed with children and adolescents who responded positively to the ISAAC questionnaire (International Study of Asthma and Allergies in Childhood) in relation to symptoms of AR and forwarded for diagnosis confirmation, through detailed clinical assessment. The sample of this study included 40 patients and was divided into two groups: a treatment group with 22 patients and a placebo group with 18 patients.

\section{Trial design and setting}

Random allocation of patients was made with a table of random numbers, using Epi Info (version 6.04). The study was carried out in a Pediatric Pulmonology Outpatient Clinic that assists exclusively patients from families covered by the Brazilian Public Health System.

\section{Criteria for inclusion and exclusion}

We included AR patients aged between 8 and 15, diagnosed in accordance with Allergic Rhinitis and its Impact on Asthma (ARIA) ${ }^{(12)}$ definitions and confirmed through an allergy test. We excluded patients who had received intranasal corticosteroids, topical or systemic vasoconstrictors and/or cromolyn sodium, antihistamines, leukotriene receptor antagonists and specific immunotherapy in the four weeks prior to the study, patients with clinical diagnosis of upper respiratory tract infections, and patients with nasal polyps and/or a deviated septum.

\section{Definitions}

Classification of ARseverity was based on the criteria of Wilson and collaborators ${ }^{(7)}$, who described a score system in which each of six signs/symptoms (nasal obstruction, runny nose, sneezing, nasal itching, oropharyngeal itching and ocular itching) was given a score from 0 to 3 , in accordance with intensity: $\mathbf{0}$ means the absence of a certain sign/symptom; $\mathbf{1}$, when a sign/symptom was light, well tolerated and didn't interfere with the sleep or daily activities of the individual; $\mathbf{2}$, when the sign/symptom assessed caused discomfort and interfered only in activities that required high levels of concentration; and $\mathbf{3}$, when the sign/symptom assessed was of such an intensity and strength that it prevented the performance of daily activities. The points were then tallied, varying from 0 to 18. A total score between 1-6 indicated slight allergic rhinitis; between 7 and 12, moderate rhinitis; and between 13-18, severe allergic rhinitis.

\section{Follow-up}

After admission, the patients were monitored for 10 weeks with assessments by clinical score and PNIF measurement every 2 weeks. In the first assessment (time 0 ), the sample was divided into two groups in a double blind manner: the treatment group composed of patients medicated with fluticasone propionate nasal spray at $50 \mu \mathrm{g} / \mathrm{dose}(100 \mu \mathrm{g} /$ day $)$ and the placebo group treated with sodium chloride at $0.9 \%$, once a day. In the eighth week, corresponding to the fifth assessment, treatments were discontinued with reassessment after two weeks. Functional assessment by PNIF followed these recommendations: initially, the patient performed routine nasal hygiene, gently blowing the nose. With the individual standing up, the facial mask was carefully put on, whereupon the patient was instructed, from a residual volume, to vigorously breathe in through the nose with the mouth closed until total lung capacity was reached. The equipment used was the in-check-inspiratory flow meter (Clement Clarke, Harlow, England). At least three verifications were carried out, with the highest value being considered for analysis.

For the PNIF measurements recorded, the percentages predicted for age (50th percentile) were calculated according to the PNIF reference curves, proposed by lbiapina and collaborators (8).

\section{Statistical analysis}

Confidence intervals of $95 \%$ (Cl 95\%) were calculated for the means and percentages, as measures to describe the results of the variables studied ${ }^{(13)}$. Variance Analysis based on a planning of Repeated Measurements (ANOVA) was used for the assessment of treatment with nasal corticosteroids on the variation of PNIF percentage measurements in relation to predicted PNIF and on the variation of clinical AR score, in the times 0 (first evaluation) to 5 (week 10). When the analysis indicated a significant influence of one or more factors, the Multiple Comparison of Means Test - Least Significant Difference (LSD) was used to evaluate this effect. All results had a significance level of $5 \%$ ( $p$ $<0.05)$.

\section{Ethical considerations}

The trial protocol and informed consent agreement were approved by the Research Ethics Committee of Minas Gerais Federal University.

\section{Results}

The sample treatment group was composed of $72.7 \%$ male $(16 / 22)$, with a mean age of 11.3 years, and a placebo group also mostly male at $61.1 \%(11 / 18)$ and mean age of 11.9 years.

Figure 1 shows the variations in mean PNIF percentage values throughout follow-up in treatment and placebo groups. The treatment group had mean PNIF percentages higher than the placebo group, in relation to predicted value; in the placebo group, there was no difference between the six periods studied.

There was a statistically significant influence of the group $(F=421.3 ; p<0.001)$, the time $(F=7.1 ; p<0.001)$ and also the 


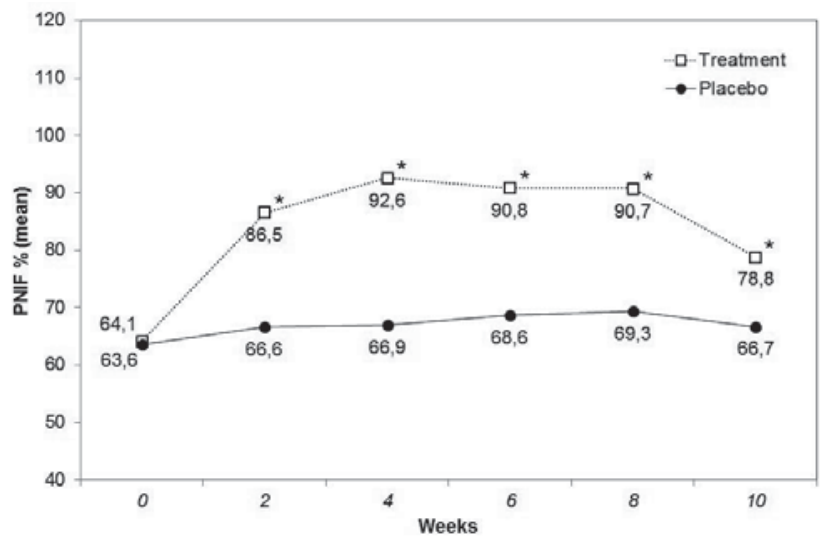

Figure 1. Variations in mean PNIF percentages values throughout follow-up in treatment and placebo groups.

interaction between time and group $(F=3.8 ; p=0.006)$ on PNIF percentage results.

In relation to the clinical score, Figure 2 shows that the treatment determinates decay in its measurements. There was a statistically significant influence between the groups $(F=26.1$; $\mathrm{p}<0.001)$, the time $(F=47.0 ; \mathrm{p}<0.001)$ and also the interaction between time and group $(F=24.2 ; p<0.001)$ on the clinical score results. However, in the placebo group there was no difference $(p>0.05)$ between the six periods of observation. It is noteworthy that PNIF percentage values do not return to the values before the intervention, in contrast with clinical score values.

\section{Discussion}

The current study showed evidence of the usefulness in applying the PNIF value reference curves, since a significant increase in PNIF percentage values was found after the introduction of intranasal corticosteroid, from the 2nd week onwards, suggesting improvement in nasal obstruction. Apart from this, its removal after 8 weeks of use was accompanied by a reduction in PNIF values. It should be highlighted that the PNIF percentages accompanied the clinical scores, indicating improvement in nasal obstruction, with the exception of the assessment at time 5 (week 10), in which clinical scores appear unable to characterize the residual effect of the corticosteroid (patient quickly refers return of AR symptoms). In the placebo group, the PNIF percentage values and the clinical scores remain unaltered throughout follow-up. There was a statistically significant difference with regard to PNIF and the clinical score with the use of nasal corticosteroid, confirming its effectiveness in the control of $A R^{(14,15)}$. It is known that PNIF may be used as an instrument for objective assessment of AR treatment ${ }^{(1)}$, since clinical examination is not effective to evaluate the patient in the presence of nasal

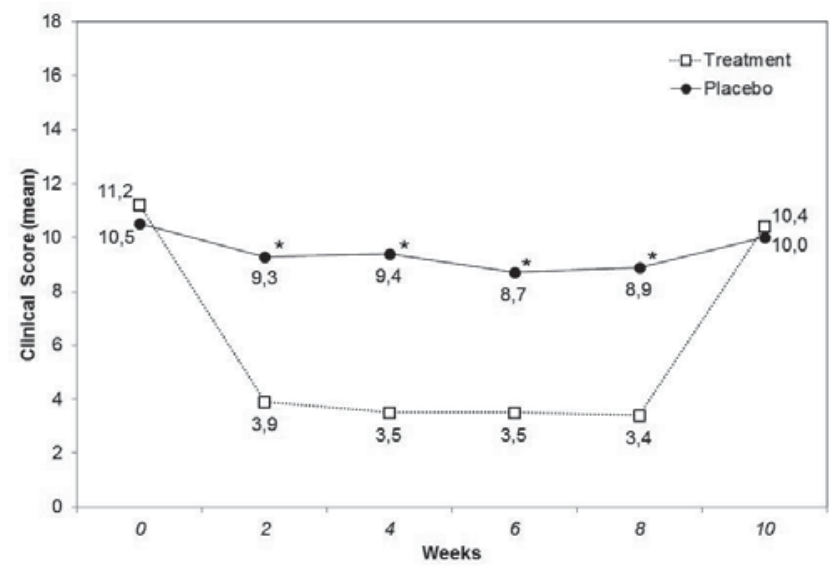

Figure 2. Variations in mean clinical scores throughout follow-up in treatment and placebo groups obstruction. Gomes and collaborators assessed 52 patients in the pediatric agegroup and verified a weak correlation between AR symptoms and PNIF ${ }^{(7)}$. Therefore, this measure has become a useful tool in helping in the education of patients, particularly those adapted to their chronic symptoms, thereby helping in adherence to treatment and improving the quality of life (16). PNIF has been used to objectively quantify one of the most relevant clinical findings, which is, at the same time, the most troublesome in the assessment and quantification of $A R$, namely, nasal obstruction, and the growing acceptance of PNIF arises from its simplicity, low cost and availability, allowing for its use in clinics ${ }^{(16)}$. According to Ottaviano et al., who carried out a study on an adult population of 137 patients, it was considered a useful method in the assessment of nasal patency in primary and secondary health care, aiding in the diagnosis of nasal disease ${ }^{(17)}$. Another important study was carried out by Chaves et al., who investigated 297 healthy children and adolescents between 6 and 18 years of age. A positive correlation between PNIF and gender, age, height/weight percentile and Peak Expiratory Flow (PEF) was found and also a moderate correlation between PNIF and PEF ( $r=0.433 ; p \leq 0.001)$ leading them to conclude that the PEF is predictive and related to the PNIF value in healthy children ${ }^{(18)}$. Ninety seven adult volunteers were studied by Ottaviano et al. with measurement of unilateral PNIF suggesting that it could become an easy method to assess septum deviation or any case where there is suspicion of single nostril occlusion ${ }^{(19)}$.

Until now, no study has been found in the literature that evaluates the PNIF reference curves in the follow-up of patients with AR treated with nasal corticotherapy. This strategy appears important given that the use of curves optimizes assessment, it is an easily employable tool, and may be used in the follow-up of children with AR, similar to the pulmonary function predicted 
values in patients with asthma. Therefore, this study envisages a greater usage of the curve in AR patients.

Other studies could be carried out with a sample composed of adults. Besides this, it wou ld be interesting if other studies, similar to this one, are performed with the application of reference curves for Greek and Dutch populations ${ }^{(10,11)}$ to serve as a comparison with the current study and, probably, to reinforce the idea that this instrument is useful in clinical practice. Moreover, it is suggested that studies should be done on comparisons of PNIF percentage values with other objective measurements, such as acoustic rhinomanometry and acoustic rhinometry.

The study was the first that used a PNIF reference curve in the longitudinal follow-up of children and adolescents with AR and was composed of a relatively small number of patients, which could restrict the generalization of results. Nevertheless, based on these results found, it cannot be ruled out that the findings of our study may be extrapolated even with the use of other re- ference curves and this should certainly be the subject of future investigations. In conclusion, PNIF reference values, apart from being important in the description of nasal obstruction, can be a useful parameter in monitoring children and adolescents in AR treatment.

\section{Authorship contribution}

SdSF: conceptualisation and design of the study, the acquisition, analysis and interpretation of data, statistical analysis, and the drafting of the paper. CRdA: conceptualisation and design of the study, the analysis and interpretation of data, statistical analysis, and the drafting of the paper. $\mathrm{CdCl}$ : conceptualisation and design of the study, the analysis and interpretation of data, statistical analysis, and the drafting of the paper. $\mathrm{CdCl}$ also supervised the study. All authors contributed to the critical revision of the paper and approved the final manuscript for publication.

\section{Conflict of interest}

There are no competing interests for any of the authors.

\section{References}

1. Bousquet J, Schünemann HJ, Samolinski $B$, et al. Allergic Rhinitis and its Impact on Asthma (ARIA): achievements in 10 years and future needs. J Allergy Clin Immunol. 2012; 130: 1049-1062.

2. ISAAC Phases One and Three repeat multicountry cross-sectional surveys. Lancet. 2006; 368( 9537): 733-743.

3. Aït-Khaled N, Pearce N, Anderson HR, et al. Global map of the prevalence of symptoms of rhinoconjunctivitis in children: The International Study of Asthma and Allergies in Childhood (ISAAC) Phase Three. Allergy. 2009; 64: 123-148.

4. Solé D, Cassol VE, Silva AR, et al. Prevalence of symptoms of asthma, rhinitis, and atopic eczema among adolescents living in urban and rural areas in different regions of Brazil. Allergol Immunopathol (Madr). 2007; 35: 248253.

5. Solé D, Wandalsen GF, Camelo-Nunes IC, Naspitz CK, ISAAC-Brazilian group. Prevalence of symptoms of asthma, rhinitis and atopic eczema among Brazilian children and adolescents identified by the International Study of Asthma and Allergies in Childhood (ISAAC)-Phase 3. J Pediatr . 2006; 82: 341-346.

6. Castro LK, Cerci Neto A, Ferreira Filho OF. Prevalence of symptoms of asthma, rhinitis and atopic eczema among students between 6 and 7 years of age in the city of Londrina, Brazil. J Bras Pneumol. 2010; 36: 286-292.

7. Wilson AM, Dempsey OJ, Sims EJ, Lipworth BJ. A comparison of topical budesonide and oral montelukast in seasonal allergic rhinitis and asthma. Clin
Exp Allergy. 2001; 31: 616-624.

8. Ibiapina CC, Andrade CR,Camargos PAM, Alvim CG, Cruz AA. Reference values for peak nasal inspiratory flow in children and adolescents in Brazil. Rhinology. 2011; 49: 304-308.

9. Dor-Wojnarowska A, Rabski M, Fal AM, et al. An attempt to estimate parameters useful for establishing a normal range for peak nasal inspiratory flow. Pneumolol Alergol Pol. 2011: 79: 320-325.

10. Papachristou A, Bourli E, Aivazi D, et al. Normal peak nasal inspiratory flow rate values in Greek children and adolescents. Hippokratia. 2008; 12: 94-97.

11. van Spronsen E, Ebbens FA, Fokkens WJ. Normal peak nasal inspiratory flow rate values in healthy children aged 6 to 11 years in the Netherlands. Rhinology. 2012, 50: 22-25.

12. Brozek JL, Bousquet J, Baena-Cagnani CE, et al. Allergic Rhinitis and its Impact on Asthma (ARIA) guidelines: 2010 revision. J Allergy Clin Immunol. 2010; 126: 466-476.

13. Milliken GA, Johnson DE. Analysis of Messy Data. New York: Chapman \& Hall, 1992, 472.

14. Weiner JM, Abramson MJ, Puy RM. Intranasal corticosteroids versus oral $\mathrm{H} 1$ receptor antagonists in allergic rhinitis: systematic review of randomised controlled trials. BMJ. 1998; 317: 1624-1629.

15. Jen A, Baroody F, de Tineo M, Haney L, Blair C, Naclerio R. As-needed use of fluticasone propionate nasal spray reduces symptoms of seasonal allergic rhinitis. J Allergy Clin Immunol. 2000; 105: 732738.

16. Gomes DL, Camargos PAM, Ibiapina CC,
Andrade CR. Nasal peak inspiratory flow and clinical score in children and adolescents with allergic rhinitis. Rhinology. 2008; 46: 276-280.

17. Ottaviano G, Scadding GK, Coles S, Lund VJ. Peak nasal inspiratory flow; normal range in adult population. Rhinology. 2006; 44: 32-35.

18. Chaves C, Ibiapina CC, de Andrade CR, Godinho R, Alvim CG, Cruz AA. Correlation between peak nasal inspiratory flow and peak expiratory flow in children and adolescents. Rhinology. 2012; 50: 381-385.

19. Unilateral peak nasal inspiratory flow, normal values in adult population. Ottaviano G, Scadding GK, Scarpa B, Accordi D, Staffieri A, Lund VJ. Rhinology. 2012; 50: 386-392.

Cássio da Cunha Ibiapina

Departamento de Pediatria da

Faculdade de Medicina

Universidade Federal de Minas

Gerais

Avenida Professor Alfredo Balena,

190 / Sala 267

30130-100 Belo Horizonte

Brazil

Tel: + 55-31-34099772 